\title{
A Joint Influence of Nitrogen Doping and Oxygen Vacancy on the Manganese Dioxide as a High- Capacity Cathode for Zinc-Ion Battery
}

Zining Zhang, Song $\mathrm{Li}^{*}$, Bin Zhao, Xiaole Zhang, Xinyu Wang, Zhongsheng Wen, Shijun Ji and Juncai Sun

Institute of Materials and Technology, Dalian Maritime University, Dalian 116026, China

1 Results of XRD, EDS mappings and FTIR spectra

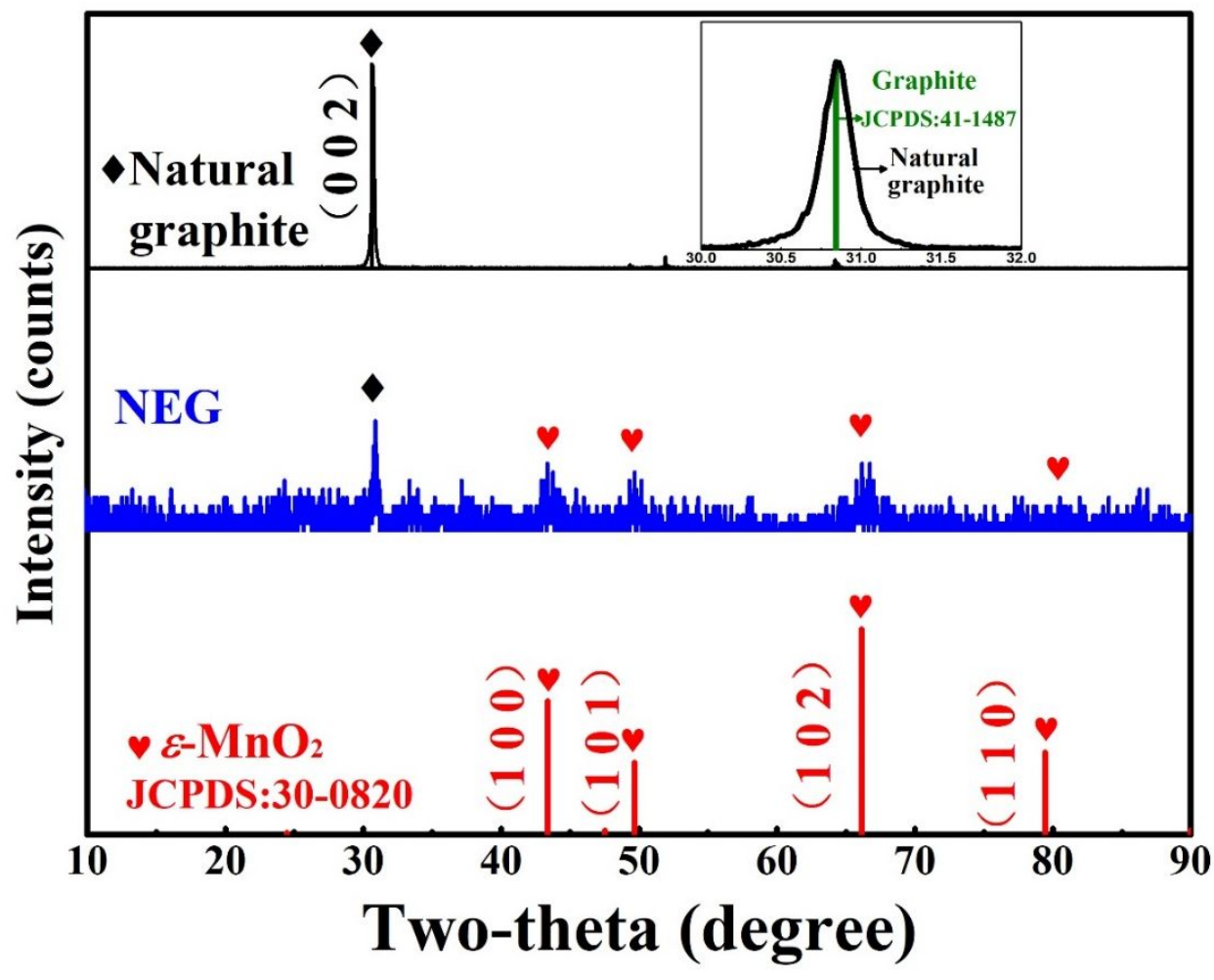

Figure S1. XRD pattern image of NEG.

${ }^{*}$ Corresponding author at: Department of Materials, Dalian Maritime University, No.1 Linghai Road, Gaoxin District, Dalian 116026, China. E-mail address: lisong@dlmu.edu.cn . Tel: +86411-84727971. Fax: +86-411-84727971 


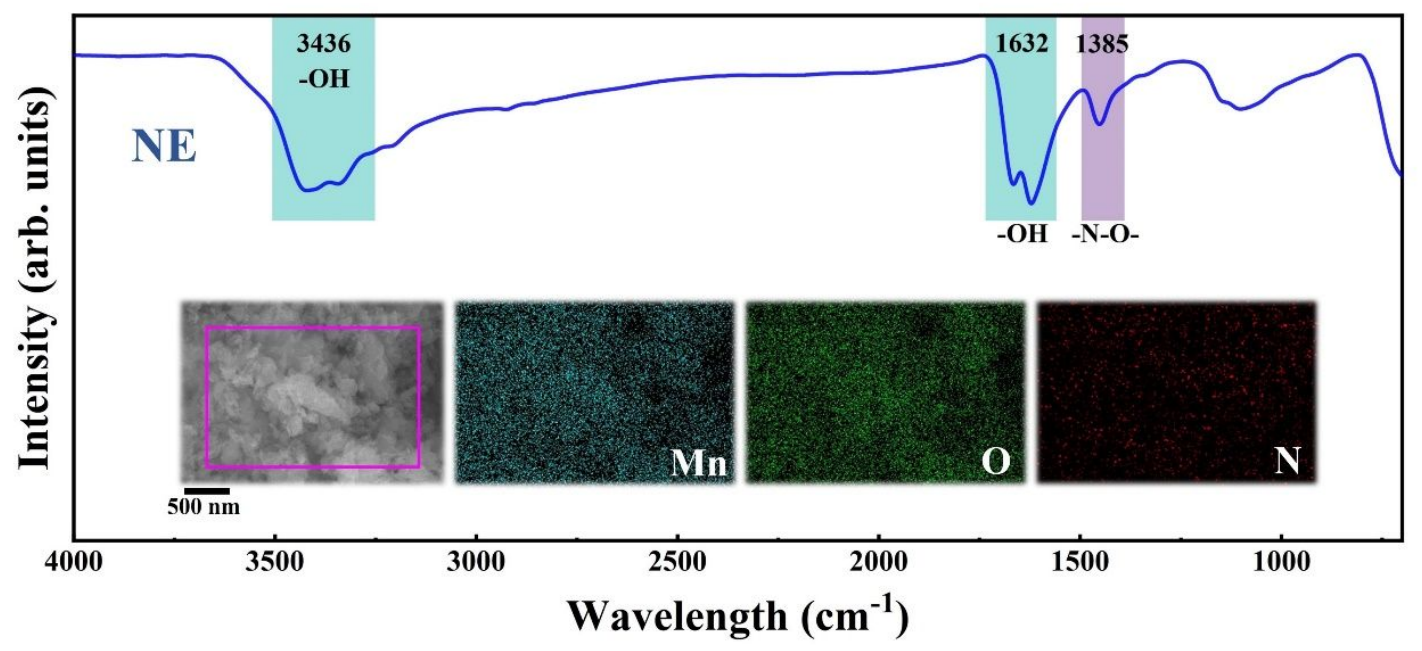

Figure S2. FTIR spectra and EDS mappings of NE.

2 Cycling performance of $\mathrm{NE}_{30} \mathrm{G}_{10}, \mathrm{NE}_{30} \mathrm{G}_{40}$ and $\mathrm{NE}_{30} \mathrm{G}_{60}$

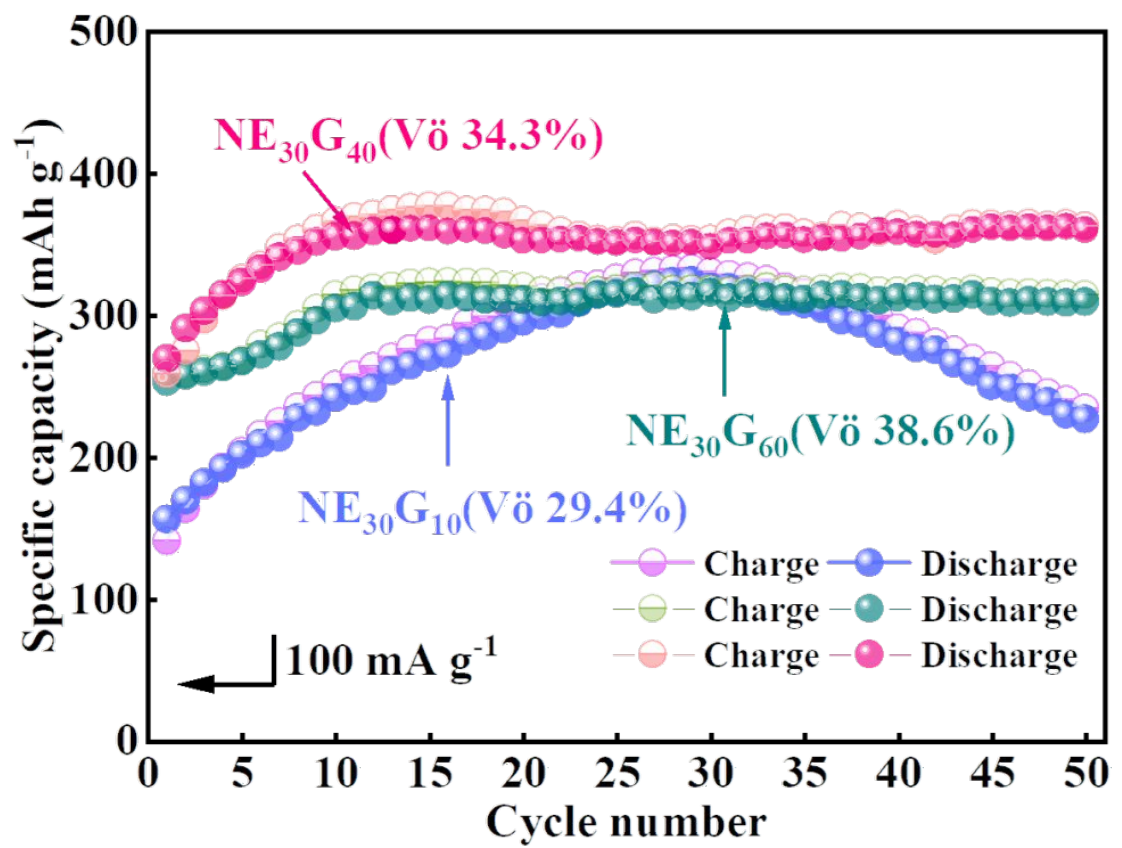

Figure S3. Cycling performance of $\mathrm{NE}_{30} \mathrm{G}_{10}, \mathrm{NE}_{30} \mathrm{G}_{40}$ and $\mathrm{NE}_{30} \mathrm{G}_{60}$ at $100 \mathrm{~mA} \mathrm{~g}{ }^{-1}$.

\section{Charge-discharge GITT curves}




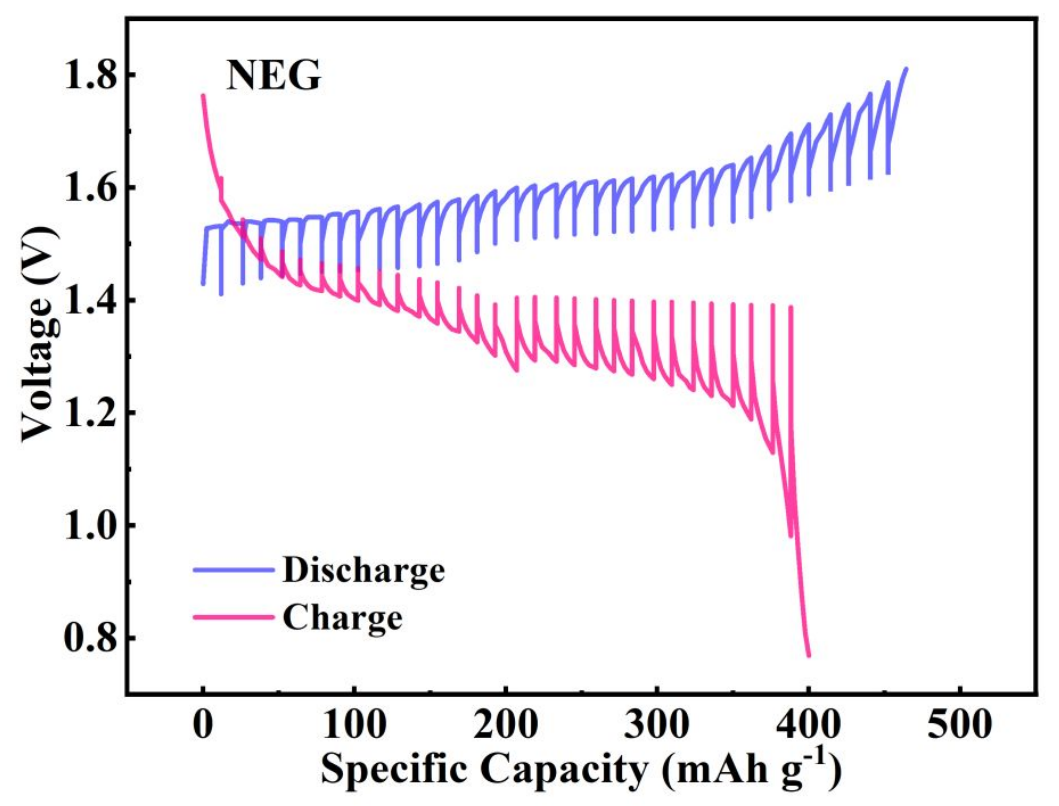

Figure S4. Charge-discharge GITT curves for NEG at a current density of $200 \mathrm{~mA} \mathrm{~g}^{-1}$.

\section{The calculation process of $\mathrm{CV}$ and GITT}

$\mathrm{CV}$ was employed to investigate the storage mechanism and reaction kinetics in NEG. Apparently, with the increase of scan rates, the peaks in CVs gradually grow broad, but the shapes of CVs maintain affinity. To gain insight into the storage mechanism of electrode, there is a typical way of analyzing the $\mathrm{CV}$ data at various sweep rates according to the following equation: ${ }^{1}$

$$
\stackrel{p}{\imath}=a v^{b} \#(1)
$$

where the measured current $\mathrm{i}$ obeys a power law relationship with the sweep rate $\mathrm{v}$. Both $\mathrm{a}$ and $\mathrm{b}$ are adjustable parameters, with $\mathrm{b}$ values determined from the slope of the plot of $\log$ i versus $\log \mathrm{v}$, as described in the following equation:

$$
\log i=\log a+b \log v \#(2)
$$

the coefficient $b$ varies in the range of $0.5-1.0$, so there are two well-defined conditions, namely, $b=0.5$ and $b=1.0$. The $b$ value of 0.5 is indicative of a diffusioncontrolled insertion process, while the $b$ value of 1.0 is representative of a surface capacitive process. ${ }^{2}$ As shown in Figure S7, the b values of three redox peaks are calculated as 0.519 (peak 1), 0.523 (peak 2) and 0.521 (peak 3), indicating that the electrochemical kinetic of NEG electrode is related to both diffusion-controlled process 
and capacitive effects, but the diffusion-controlled behavior is the dominant process. (Figure S3)To better know the contributions to the total capacity of NEG electrode, the current response (i) at a fixed potential (V) can be expressed as the combination of two separate mechanisms, namely, surface capacitive effects and diffusion-controlled insertion, as described below: ${ }^{3}$

$$
\stackrel{a}{\sharp}(v)=k_{1} v+k_{2} v^{\frac{1}{2}} \#(3)
$$

where the $\mathrm{k}_{1} \mathrm{v}$ and $\mathrm{k}_{2} \mathrm{~V} 1 / 2$ correspond to the current contributions arising from the surface capacitive effects and the diffusion-controlled insertion processes, respectively. Specifically, the diffusion-controlled process holds the dominant position in the total contribution of NEG electrode under low current densities, while the capacitive behavior contributes most under high current densities.

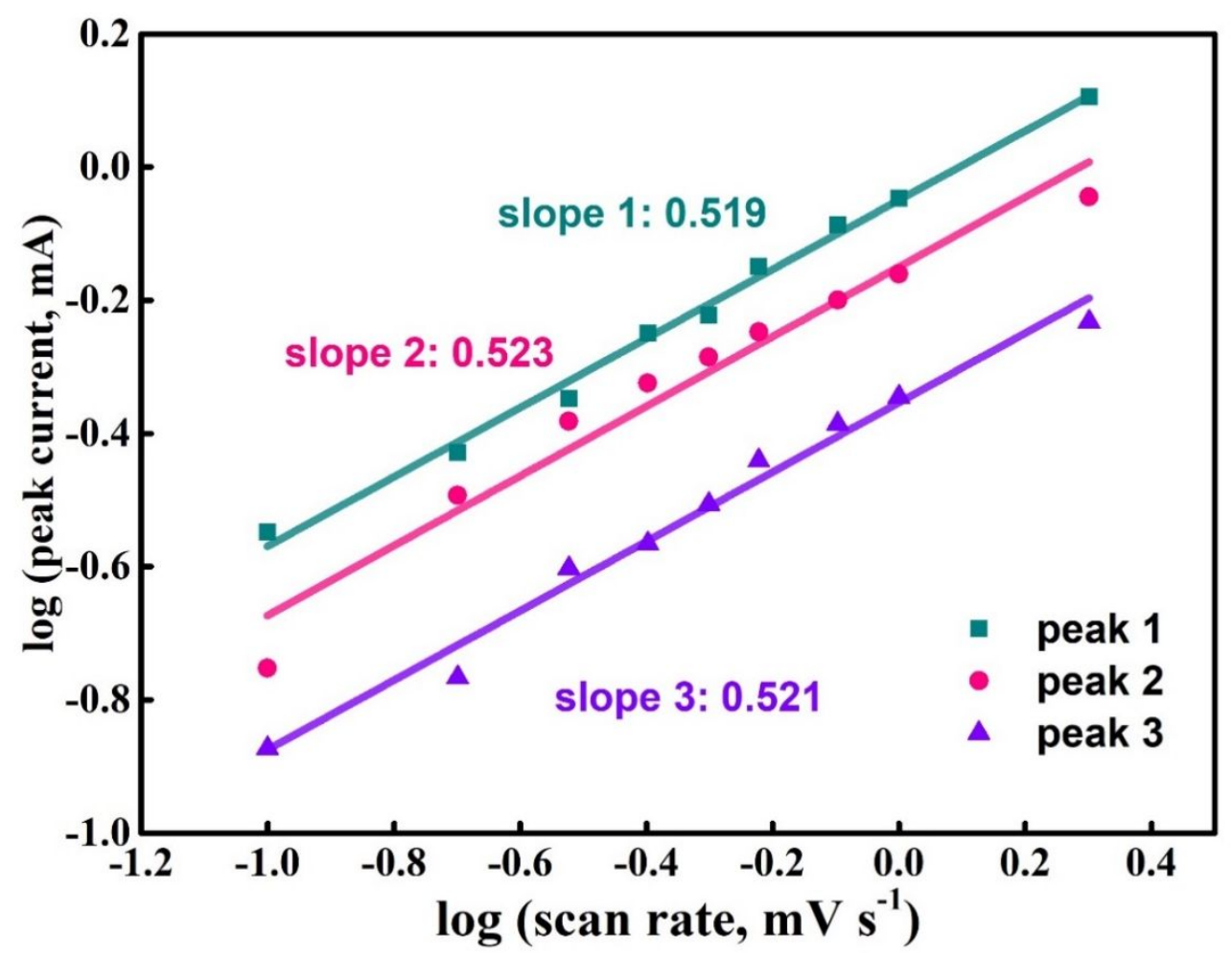

Figure S5. Log i and $\log \mathrm{v}$ plots at specific peak currents.

The GITT was employed to determine the thermodynamic voltage-composition relationship, where we could find out the corresponding ion diffusion coefficients in 
NEG cathode. In our GITT study, a cell was charged or discharged at $200 \mathrm{~mA} \mathrm{~g}^{-1}$ rate for $20 \mathrm{~min}$, followed by a $1 \mathrm{~h}$ open circuit step to allow relaxation back to equilibrium (defined as $\mathrm{dE} / \mathrm{dt}<0.2 \mathrm{mV} \mathrm{h}^{-1}$ ) (Figure S4). The procedure was continued until the charge (or discharge) voltage reached $1.8 \mathrm{~V}(0.8 \mathrm{~V})$. The ion diffusion coefficients could be calculated using the following equation first outlined by Weppner and Huggins: ${ }^{4}$

$$
D=\frac{4}{\pi}\left(I \frac{V_{m}}{Z_{A F S}}\right)^{2}\left(\frac{d E / d \delta}{d E / d \sqrt{\mathrm{t}}}\right)^{2}
$$

where I is the current (A); Vm is the molar volume of the NEG $\left(\mathrm{cm}^{3} \mathrm{~mol}^{-1}\right)$; ZA is the charge number; $\mathrm{F}$ is the Faraday's constant (96485 $\left.\mathrm{C} \mathrm{mol}^{-1}\right)$; $\mathrm{S}$ is the electrode/electrolyte contact area $\left(\mathrm{cm}^{2}\right) ; \mathrm{dE} / \mathrm{d} \delta$ is the slope of the coulometric titration curve, found by plotting the steady state voltages E (V) measured after each titration step $\delta ; \mathrm{dE} / \mathrm{d} \sqrt{t}$ is the slope of the linearized plot of the potential $\mathrm{E}(\mathrm{V})$ during the current pulse of duration $\mathrm{t}(\mathrm{s})$. If sufficiently small currents are applied for short time intervals, so that $\mathrm{dE} / \mathrm{d} \sqrt{t}$ can be considered linear and the coulometric titration curve can be also considered linear over the composition range involved in that step, the above equation can be simplified into: ${ }^{5}$

$$
D=\frac{4}{\pi^{\tau}}\left(\frac{\mathrm{n}_{\mathrm{m}} V_{\mathrm{m}}}{S}\right)^{2}\left(\frac{\Delta E_{\mathrm{s}}}{\Delta E_{\tau}}\right)^{2}
$$

Here, $\tau$ is the duration of the current pulse (s); $\mathrm{nm}$ is the number of moles (mol); $\mathrm{Vm}$ is the molar volume of the electrode $\left(\mathrm{cm}^{3} \mathrm{~mol}^{-1}\right) ; \mathrm{S}$ is the electrode/electrolyte contact area $\left(\mathrm{cm}^{2}\right) ; \Delta$ Es is the steady-state voltage change, due to the current pulse and $\Delta \mathrm{Et}$ is the voltage change during the constant current pulse, eliminating the iR drop.

\section{References}

1. Lindstrom, H.; Sodergren, S.; Solbrand, A.; Rensmo, H.; Hjelm, J.; Hagfeldt, A.;Lindquist, S.-E. $\mathrm{Li}^{+}$Ion Insertion in $\mathrm{TiO}_{2}$ (Anatase). 2. Voltammetry on Nanoporous Films. J. Phys. Chem. B 1997, 101, 7717-7722.

2. Wang, J.; Polleux, J.; Lim, J.;Dunn, B. Pseudocapacitive Contributions to Electrochemical Energy Storage in $\mathrm{TiO}_{2}$ (Anatase) Nanoparticles. J. Phys. Chem. C 2007, 111, 14925-14931. 
3. Ali, G. A. M.; Yusoff, M. M.; Shaaban, E. R.;Chong, K. F. High Performance $\mathrm{MnO}_{2}$ Nanoflower Supercapacitor Electrode by Electrochemical Recycling of Spent Batteries. Ceram. Int. 2017, 43, 84408448.

4. Yan, M.; Mai, L.; He, P.; Chen, Y.; Shanyu Wang; Wei, Q.; Zhao, K.; Xu, X.; An, Q.; Shuang, Y.; Shao, Y.; Karl T. Mueller; Liu, J.;Yang, J. Water Lubricated Intercalation in $\mathrm{V}_{2} \mathrm{O}_{5} \cdot \mathrm{nH}_{2} \mathrm{O}$ for High Capacity and High Rate Aqueous Rechargeable Zinc Batteries. Adv. Mater. 2018, 30, 1703725-1703739.

5. Xia, C.; Guo, J.; Lei, Y.; Liang, H.; Zhao, C.;Alshareef, H. N. Rechargeable Aqueous Zinc-Ion Battery Based on Porous Framework Zinc Pyrovanadate Intercalation Cathode. Adv. Mater. 2018, 30, 1705580-1705587. 